\title{
WNT/ $\beta$-catenin increases the production of incretins by entero-endocrine cells
}

\author{
J. M. García-Martínez • A. Chocarro-Calvo • \\ C. M. Moya • C. García-Jiménez
}

Received: 17 February 2009 / Accepted: 21 May 2009/Published online: 7 July 2009

(C) Springer-Verlag 2009

\begin{abstract}
Aims/hypothesis Glucose-dependent insulinotropic peptide (GIP) plays a pivotal role in the regulation of glucose homeostasis. Rates of diet-induced obesity, insulin resistance and type 2 diabetes are decreased when GIP signalling is disturbed in mice, suggesting that GIP plays a role in the onset of type 2 diabetes. WNT signalling is linked to type 2 diabetes and induces synthesis of the other incretin, glucagon-like peptide 1 (GLP-1). GLP-1 analogues improve treatment of type 2 diabetes patients in whom GLP-1 signalling is intact and have captured clinical attention. GIP levels are altered at the onset of type 2 diabetes and later on, while GIP signalling is impaired. Thus, GIP is not a candidate for treatment but might be an important target from a prevention perspective. Hypothesising that hypersecretion of GIP links altered WNT signalling to the onset of type 2 diabetes, we sought to determine whether WNT signalling induces GIP production by entero-endocrine cells.

Methods RT-PCR and chromatin immunoprecipitation (ChIP) were used to study Gip gene induction. Gip promoter
\end{abstract}

J. M. García-Martínez and A. Chocarro-Calvo contributed equally to this work.

Electronic supplementary material The online version of this article (doi:10.1007/s00125-009-1429-1) contains supplementary material, which is available to authorised users.

J. M. García-Martínez · A. Chocarro-Calvo •

C. García-Jiménez $(\bowtie)$

Dptal I. Despacho 020, Facultad de Ciencias de la Salud,

Universidad Rey Juan Carlos,

28922 Alcorcon, Madrid, Spain

e-mail: custodia.garcia@urjc.es

C. M. Moya

Instituto de Investigaciones Biomédicas,

Consejo Superior de Investigaciones Científicas (CSIC),

Madrid, Spain elements mediating WNT/lithium induction were identified (electrophoretic mobility shift assay, co-transfection of deletion mutants, ChIP).

Results Lithium or $\mathrm{WNT} / \beta$-catenin signalling enhanced GIP production by entero-endocrine cells through a conserved site in the proximal Gip promoter. Lithium favours lymphoid enhancer factor- $1 / \beta$-catenin binding to Gip promoter and diminishes ChIP through T cell factor-4 and histone deacetylase 1.

Conclusions/interpretation Lithium and WNT are incretin inducers in general. This work provides a novel link between WNT signalling, obesity and diabetes.

Keywords $\beta$-Catenin - Chromatin - Diabetes . Glucose-dependent insulinotropic peptide · Incretin . Lithium · Obesity · WNT

$\begin{array}{ll}\text { Abbreviations } \\ \text { ChIP } & \text { Chromatin immunoprecipitation } \\ \text { EMSA } & \text { Electrophoretic mobility shift assay } \\ \text { GIP } & \text { Glucose-dependent insulinotropic peptide } \\ \text { GLP-1 } & \text { Glucagon-like peptide 1 } \\ \text { GSK3 } \beta & \text { Glycogen synthase kinase } 3 \beta \\ \text { HDAC1 } & \text { Histone deacetylase 1 } \\ \text { LEF } & \text { Lymphoid enhancer factor } \\ \text { TCF } & \text { T cell factor }\end{array}$

Introduction

Incretins are hormones that enhance insulin secretion in response to an oral glucose load. Glucose-dependent insulinotropic peptide (GIP) and glucagon-like peptide 1 (GLP-1), produced along the small intestinal epithelium, 
are the major incretins. Up to $80 \%$ of total insulin secreted after an oral load of glucose is due to incretin action $[1,2]$. GIP is a 42 amino acid peptide that has a half-life of 5 to $7 \mathrm{~min}$, and induces insulin secretion by pancreatic $\beta$ cells, triacylglycerol synthesis/storage by adipocytes and also adipokine secretion (leptin, resistin) [3]. GIP plays a major role in obesity induced by overnutrition [4-7], which often leads to type 2 diabetes. GIP function is controlled by maintaining adequate levels (synthesis and secretion) and signalling through its specific receptor, GIP receptor (GIPR). Gipr $^{-/-}$mice exhibit severe glucose intolerance after an oral glucose load [8]. Long-term GIP signalling inhibition protects $\mathrm{Gipr}^{-/-}$mice from age-associated insulin resistance [9]. Compared with controls, $\mathrm{Gipr}^{-/}$mice exposed to chronic nutritional excess do not become obese [10]. Indeed, GIP has now become a potential target for anti-obesity and anti-diabetes drugs [11]. Strong association of Tcf7l2 variants (effectors of WNT signalling) with type 2 diabetes established a genetic linkage between diabetes and WNT signalling (see Welters et al. [12] and references therein). Tcf7l2 is a T cell factor (TCF)/lymphoid enhancer factor (LEF) that regulates adipogenesis and GLP-1 production, suggesting a link with incretin action [12]. $\mathrm{TCF} / \mathrm{LEF}$ factors repress transcription by targeting corepressors such as histone deacetylase 1 (HDAC1), to their binding site. $\beta$-Catenin binding converts TCF/LEF factors into potent activators, displacing co-repressors and recruiting histone acetylases and co-activators. $\beta$-Catenin enters the nucleus following cytoplasmic accumulation when the priming enzyme for its ubiquitination and degradation, glycogen synthase kinase $3 \beta$ (GSK3 3 ), is inhibited (for reviews see Clevers [13] and Roose and Clevers [14]). WNT signalling leads to GSK3 $\beta$ inhibition, soluble $\beta$ catenin accumulation and TCF/LEF-dependent transcriptional activation $[13,14]$. Complexity and instability of WNT proteins often led to use of $\mathrm{LiCl}$ to inhibit GSK3 $\beta$ mimicking of WNT signalling $[15,16]$. LiCl increased GLP-1 production in entero-endocrine GLUTag cells [17, 18] through a TCF/LEF site in the rat proglucagon $(G c g)$ promoter and WNT was later shown to be an inducer and effector of GLP-1 [19]. Transcriptional control of Gip expression is poorly understood [20-23]. We investigated whether WNT signalling controls Gip expression, promoting incretin production in general. This is important because although WNT is known to be critical for development and maintenance of gut epithelial cells [24, 25], its role in entero-endocrine differentiation remains obscure. We identified a functional TCF/LEF binding site, which is strongly conserved in mouse, rat and humans, and regulated by $\mathrm{WNT} / \beta$-catenin and lithium in mouse Gip promoter. Mouse proglucagon promoter $(G c g)$ was used as positively regulated control. We show that lithium and WNT favour $\beta$-catenin nuclear entry and targeting to fragments containing the reported TCF/LEF element. Furthermore, lithium positively selected binding of LEF1/ $\beta$ catenin to mouse Gip and Gcg promoters in enteroendocrine STC-1 cells.

\section{Methods}

Cell culture The STC-1 cell line, derived from a mouse intestinal tumour [26], is the only in vitro system known to produce and secrete GIP. Cells cultured in DMEM with $10 \% \mathrm{vol} / \mathrm{vol}$ fetal bovine serum were stimulated with $\mathrm{LiCl}$ (Sigma Chemical, St Louis, MO, USA) or recombinant purified WNT3A (Millipore, Billerica, MA, USA). Transient transfections were seeded in 24 well plates at $50 \%$ confluence using JetPei PolyPlus reagent (Genycell Biotech, Santa Fe, Granada, Spain), following the manufacturer's instructions. Luciferase reporters were transfected at $125 \mathrm{ng} /$ well and Renilla at $25 \mathrm{ng} /$ well, with expression vectors $200 \mathrm{ng} / \mathrm{well}$. Total ng of DNA transfected per well was made equal to the corresponding empty vectors. Luciferase assays were performed using a kit and dual injectors (Dual Luciferase and Luminometer Glomax 96 respectively; both Promega, Madison, WI, USA).

Constructs Mouse Gip and Gcg promoters were cloned using genomic DNA from STC-1 cells, proof-reading polymerase (Phusion; Finnzymes Oy, Keilaranta, Espoo, Finland) and specific primers (Electronic supplementary material [ESM] Table 1). PCR conditions were: $98^{\circ} \mathrm{C} 30 \mathrm{~s}$; $60^{\circ} \mathrm{C} 30 \mathrm{~s}$; and $72^{\circ} \mathrm{C} 1 \mathrm{~min}$. PCR fragments gel-purified with a kit (Qiagen, Crawley, UK) were cloned into pGL3b (Promega). For sequencing we used a sequence analyser (ABI Prism 3100 Avant; Applied Biosystems, Alcobendas, Madrid, Spain). TESS software [27] was used for in silico sequence analysis. Super8XTOPFlash and Super8XFOPflash were a gift from R. Moon (HHMI/Pharmacology, University of Washington, Seattle, WA, USA). Super8XTOPFlash contains eight copies of the sequence AGATCAAAGGgggta, including optimal TCF/LEF binding site (uppercase) and a spacer (lowercase). Bases in that sequence (AT) are substituted in the mutant Super8XFOPflash by GC. Kinase-inactive GSK3 $\beta$ contains a K85R mutation that inactivates ATP binding [28]. $\beta$-Catenin expression vector was a gift from C. Goding (Ludwig Institute for Cancer Research, Oxford University, UK).

Western blotting and electrophoretic mobility shift assay Whole-cell extracts were prepared in RIPA buffer (PBS 1\% [vol./vol.], Nonidet 0.5\% [wt/vol.], sodium deoxycholate $0.1 \%$ [wt/vol.], SDS) and nuclear extracts as described by Andrews and Faller [29]. Antibodies used were: anti total-GSK $3 \alpha / \beta$ (Biosource International, Camarillo, 
CA, USA), and anti-phospho-Ser9 (sc-11757) and anti$\beta$-catenin (sc-1496-R) (Santa Cruz Biotechnology, Santa Cruz, CA, USA). For electrophoretic mobility shift assay (EMSA) 1 pmol of Brn2 oligonucleotide bearing the consensus TCF/LEF binding site found in the promoter of transcription factor Brn-2 (also known as Pou3f2) [30] and labelled with HEX (Applied Biosystems) was incubated with $10 \mu \mathrm{g} \mathrm{STC}-1$ extracts. Competitions were with 100 pmol of specified unlabelled oligonucleotides (ESM Table 1). Results were analysed in a Typhoon imaging analyser 9210 (GE Healthcare, Piscataway, NJ, USA).

Quantitative RT-PCRs We reverse-transcribed $100 \mathrm{ng} / 1 \mu \mathrm{g}$ total RNA and amplified it 40 times $\left(94^{\circ} \mathrm{C} 1 \mathrm{~min}, 60^{\circ} \mathrm{C}\right.$ 15 s) with Power SYBR Green PCR Mastermix and primers listed in ESM Table 1. 18S rRNA primers were included as non-regulated control gene. Quantitative PCR was performed on cDNA triplicates using a sequence detection system (ABI Prism). Relative expression was calculated using the comparative cycle threshold $\left(\mathrm{C}_{\mathrm{t}}\right)$ method and expressed as $2^{-\Delta \Delta C_{t}}$ [31]. PCR efficiency was $\sim 100 \%$, estimated on standard curves using serial dilutions of cDNA mix and primer pairs for Gip, Gcg and 18S RNA. Reagents and detection systems were from Applied Biosystems, Alcobendas, Madrid, Spain.

Chromatin immunoprecipitation Confluent (60\%) STC-1 cells treated with $\pm 50 \mathrm{mmol} / \mathrm{L} \mathrm{LiCl}$ for $24 \mathrm{~h}$ were collected and cross-linked (1.1\% [vol./vol.] formaldehyde, $30 \mathrm{~min}$ at RT). Next, $2 \times 10^{7}$ cells $/ \mathrm{ml}$ were resuspended in $5 \mathrm{mmol} / \mathrm{l}$ PIPES pH 8, $85 \mathrm{mmol} / 1 \mathrm{KCl}, 0.5 \%$ [vol./vol.] NP40, $1 \times$ COMPLETE protease inhibitor cocktail (Roche, Indianapolis, IN, USA) and sonicated (Bioruptor UCD-200; Diagenode, Liège, Belgium) in $250 \mu$ l aliquots at high power (30 s sonication, $30 \mathrm{~s}$ pause for $12.5 \mathrm{~min})$. Chromatin from $1 \times 10^{6}$ to $2 \times 10^{6}$ cells was immunoprecipitated overnight with $4 \mu \mathrm{g}$ anti-LEF1(H-70) or anti-TCF4 (N-20; both from Santa Cruz Biotechnology), anti- $\beta$ catenin or anti-HDAC1 (both from Millipore) or a rabbit pre-immune serum. Antibody complexes were captured on protein A/G-coated magnetic beads (Invitrogen, San Diego). Chromatin was eluted in $1 \%$ (wt/vol.) SDS/ $100 \mathrm{mmol} / 1 \mathrm{NaHCO}_{3}$. DNA was purified using Proteinase $\mathrm{K}$ digestion. Equal amounts of purified DNA (measured $\mathrm{A}_{260}$ in nanodrop) from each immunoprecipitate were amplified (semi-quantitative 20-30 cycles or quantitative PCR) to calculate enrichment. Primer efficiency was $\sim 100 \%$ (determined with standard curves using dilutions of input chromatin). Five biological replicates were analysed. Mean \pm SEM of values obtained in samples treated with lithium were divided by values obtained in control untreated samples to obtain the ratio +lithium:-lithium that is presented.
Statistical analysis Results are presented as fold induction, mean $\pm \mathrm{SEM}$, from three biological replicas for luciferase assays, western blots and RT-PCR, or from five biological replicas for chromatin immunoprecipitation (ChIP).

Tests for significance between two sample groups were performed with Student's $t$ test. For multiple comparisons, ANOVA was used with Bonferroni's post test. Differences were considered statistically significant if $p<0.05$.

\section{Results}

WNT and lithium induce mouse Gip gene expression in entero-endocrine cells $\mathrm{LiCl}$ or recombinant purified WNT3A proteins were added to alternate plates of STC-1 cells $24 \mathrm{~h}$ before collection. Purified mRNA was analysed by quantitative RT-PCR using specific primers for mouse Gip and Gcg, the later served as positive control [17]. Figure 1 shows that both mouse Gip and Gcg mRNAs increased approximately two- to threefold, fivefold and 25fold with 20,50 and $100 \mathrm{mmol} / \mathrm{l} \mathrm{LiCl}$ respectively. We concluded that $\mathrm{LiCl}$ induces mouse Gip and Gcg expression in STC-1 entero-endocrine cells in a dose-dependent manner. Purified WNT3A induced mouse Gip and $G c g$ mRNA by six- and tenfold respectively. RT-PCR analysis of WNT receptors using primer pairs listed in ESM Table 2 revealed the presence of receptors frizzled 1 and lowdensity lipoprotein receptor-related proteins 5/6 (LRP-5/6) in entero-endocrine STC-1 cells, which are known to signal through the canonical WNT pathway (ESM Fig. 1). Lithium and WNT signalling inhibit GSK3 $\beta$ through accumulation of inactive phosphor-Ser $21 / \operatorname{Ser} 9$ GSK $3 \alpha / \beta$ [32]. Lithium also inhibits GSK3 $\beta$ competing with $\mathrm{Mg}^{2+}$ [33] and additionally has a wide range of targets, reviewed in [34-36]. Therefore, we sought to confirm that endogenous mouse Gip and $G c g$ induction in STC-1 cells was mediated by mimicking canonical $\mathrm{WNT} / \beta$-catenin pathway via GSK $3 \beta$ inhibition and not through alternative pathways (see below).

Lithium treatment mimics WNT signalling controlling bipartite $\beta$-catenin-TCF/LEF trans-activating factors in entero-endocrine STC-1 cells To determine whether enteroendocrine STC-1 cells were endowed with TCF/LEF factors controlled by WNT/ $\beta$-catenin, TOPFlash/FOPFlash luciferase reporters were transfected to specifically measure $\beta$-catenin/ TCF-regulated transcriptional function [37, 38]. WNT and lithium increased TOP:FOP activity ratio in STC-1 cells, suggesting that endogenous bipartite $\beta$-catenin-TCF/LEF factors formed as a result of GSK3 $\beta$ inhibition and $\beta$ catenin accumulation. Figure 2 shows that $\mathrm{LiCl}$ or WNT3A increased TOPFLASH but not FOPFLASH activity by 
a

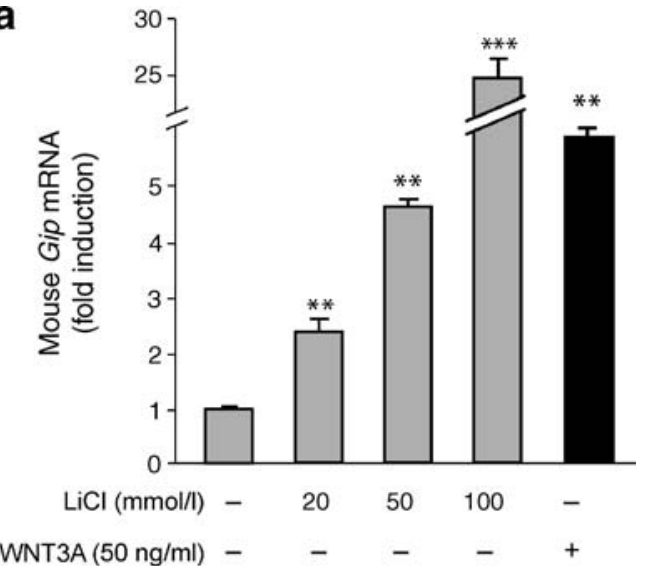

b

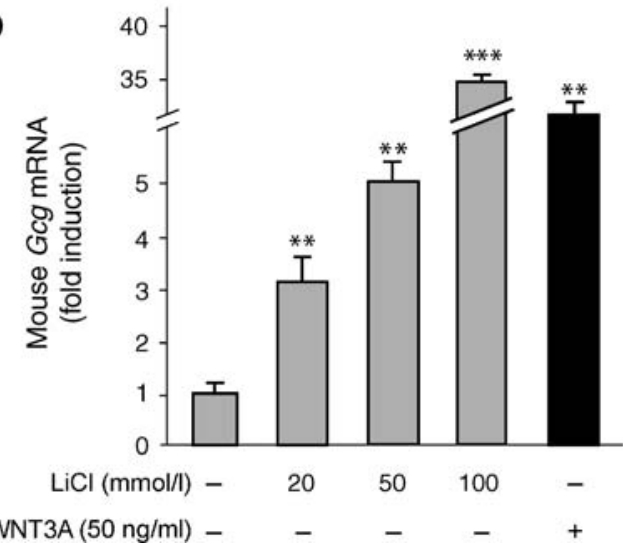

Fig. 1 Lithium and WNT induce Gip gene expression in enteroendocrine STC-1 cells. For quantitative RT-PCR, mRNAs extracted from STC-1 cells cultured for $24 \mathrm{~h}$ with 20 to $100 \mathrm{mmol} / \mathrm{l} \mathrm{LiCl}$ or recombinant WNT3A $(50 \mathrm{ng} / \mathrm{ml})$ were reverse transcribed. cDNAs were subjected to quantitative real-time PCR in the presence of fluorescent-labelled probes and primers specific for Gip (a) or Gcg $(G L P-1)$ (b) as a positive control. Values were normalised with the endogenous control (18S) and referred as fold induction over the untreated control cells. Values represent mean $\pm \mathrm{SEM} ; n=4 .{ }^{* *} p<0.01$ and $* * * p<0.001$ by ANOVA

four- to sixfold. Thus, lithium mimics WNT signalling, thereby enhancing $\beta$-catenin-TCF/LEF transcriptional activity in entero-endocrine STC-1 cells.

Lithium and WNT induce mouse Gip and Gcg promoter activities, a process reproduced by GSK3 $\beta$ inactivation The existence of cis-regulatory sequences in the mouse Gip gene which respond to $\mathrm{WNT} / \beta$-catenin signalling like the TOPFlash construct was explored by cloning mouse Gip 5'flanking regulatory regions upstream of the luciferase coding sequence. This reporter included $925 \mathrm{bp}$ of the promoter and 831 bp downstream containing the $5^{\prime}$ untranslated region and first intron. This construct or the empty reporter was transfected into STC-1 entero-endocrine cells; $\mathrm{LiCl}$ or WNT3A were added $24 \mathrm{~h}$ before collection for luciferase assays. Since the rat promoter directing the synthesis of GLP-1 (Gcg) is regulated by lithium/WNT through an element conserved in mouse [17], we cloned the mouse Gcg promoter which directs synthesis of GLP-1 and used it as positive control. Figure $3 \mathrm{a}$ shows that both lithium and WNT3A induced mouse Gip and Gcg promoter activities by about twofold. These results suggest that mouse Gip and Gcg promoters contain equivalent cis-element(s) regulated by lithium/WNT. If mouse Gip promoter induction was exerted through inhibition of GSK $3 \beta$, overexpression of a kinase inactive version of GSK3 $\beta$ (kinase-inactive GSK3 $\beta$ ) [28] should mimic lithium/WNT3A induction. In an environment loaded with excess of kinase-inactive GSK $3 \beta$, lithium should become unable to further induce mouse Gip promoter activity. Figure 3b shows that kinaseinactive GSK $3 \beta$ induced approximately twofold mouse Gip promoter activity; neither lithium nor WNT3A further increased this induction. We concluded that mouse Gip promoter contains cis-regulatory sequences controlled by lithium and WNT through GSK3 $\beta$ inactivation.

$W N T$ and lithium increase soluble $\beta$-catenin and $\beta$-catenin induces Gip promoter activity in entero-endocrine cells WNT/lithium-dependent GSK3 $\beta$ inactivation should increase soluble $\beta$-catenin and transcriptional activity of bipartite $\beta$-catenin-TCF/LEF factors on mouse Gip promoter. Figure $4 \mathrm{a}$ shows that $\mathrm{LiCl}$ and WNT significantly increased nuclear $\beta$-catenin (two- to threefold) in STC-1 cells, with quantification of three experiments and repre-

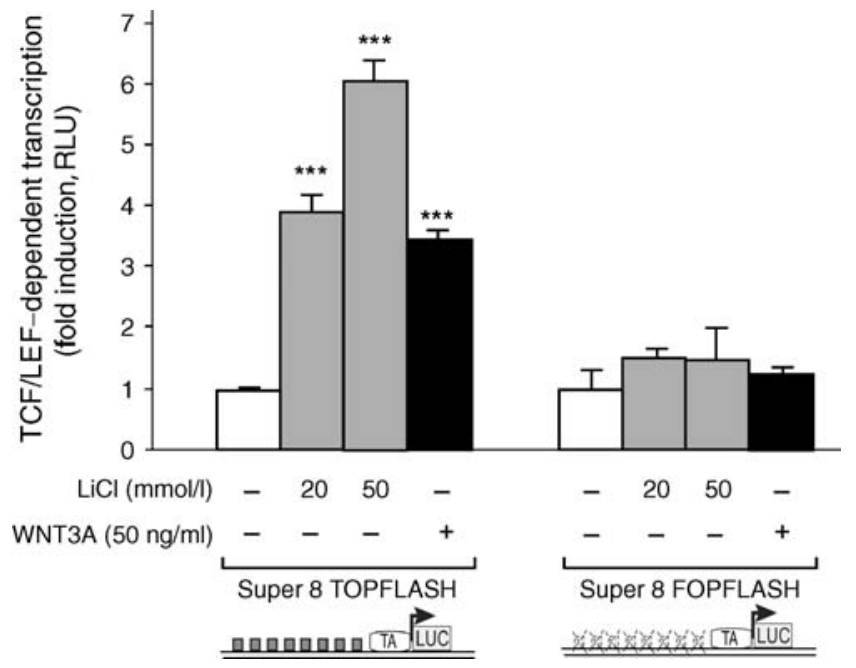

Fig. 2 Lithium activates WNT effectors such as $\beta$-catenin-TCF/LEF trans-acting factors, in entero-endocrine STC-1 cells. STC-1 cells transfected with TOPFLASH containing eight copies of the consensus TCF/LEF binding site (grey squares, $x$-axis label) or FOPFLASH (mutated version) were cultured for $24 \mathrm{~h}$ with the indicated $\mathrm{LiCl}$ or WNT3A concentration before collection for luciferase assays. Relative luciferase units (RLU) were calculated as fold induction relative to the corresponding control (mean $\pm \mathrm{SEM}, n=3$ ). ${ }^{* * *} p<0.0001$ by one-way ANOVA. No significant differences were found in the FOPFLASHtransfected cells. 


\section{a}

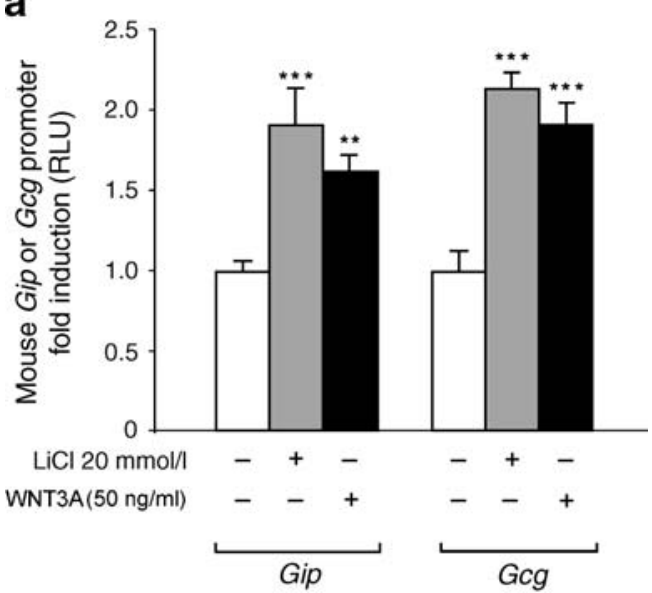

b

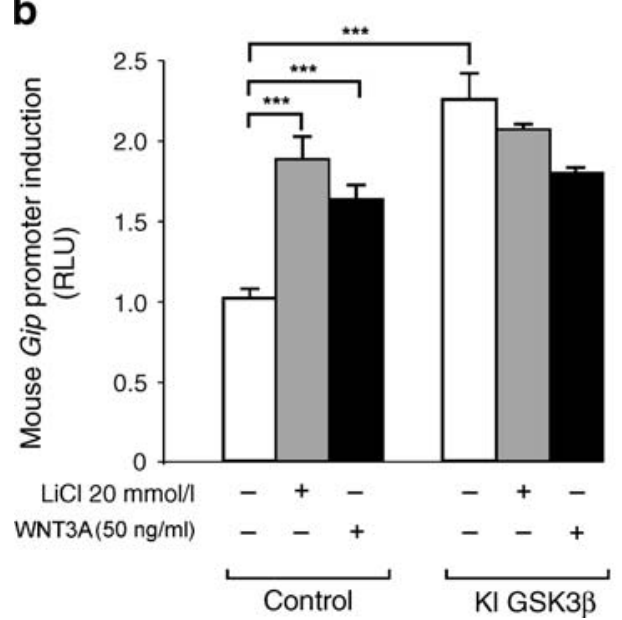

Fig. 3 Lithium and WNT induce mouse Gip and Gcg promoter activities at the same level, a process reproduced by GSK3 $\beta$ inactivation. a Lithium and WNT3A induced an approximately twofold increase of mouse Gip and Gcg promoter activity in enteroendocrine STC-1 cells. Mouse Gip reporter included 925 bp of the mouse Gip promoter and $831 \mathrm{bp}$ containing the first intron. Mouse Gcg construct contained 1921 bp of the proglucagon promoter. b Overexpression of kinase inactive GSK3 $\beta$ (KI GSK $3 \beta$ ) induced an approximately twofold increase of mouse Gip promoter activity in $24 \mathrm{~h}$ in the absence of lithium. GSK3 $\beta$ inhibition may mediate the effects of WNT and lithium on mouse Gip promoter because neither WNT3A nor lithium could increase induction when KI GSK3 $\beta$ was overproduced. Relative luciferase units (RLU) were calculated as fold induction relative to a corresponding control (mean $\pm \mathrm{SEM}, n=3$ ). ${ }^{* *} p$ $<0.01$ and $* * * p<0.001$ by Student's $t$ test

sentative western blots also showing lithium/WNT-dependent inactivation of GSK3 $\beta$ (Phospho-Ser 9) and accumulation of cytoplasmic $\beta$-catenin. Thus, lithium and WNT inactivated GSK3 $\beta$ in STC-1 cells allowing $\beta$-catenin nuclear entry. Figure $4 \mathrm{~b}$ shows that $\beta$-catenin accumulation mediated mouse Gip promoter activity increases in STC-1 cells in co-transfection experiments with the mouse Gip reporter and a vector to overexpress $\beta$-catenin. The positive control, kinase-inactive GSK $3 \beta$ and $\beta$-catenin, increased mouse Gip promoter activity some fivefold in $36 \mathrm{~h}$. Thus, lithium and WNT jointly achieve GSK3 $\beta$ inhibition and accumulation of soluble $\beta$-catenin targeting cis-regulatory elements at the mouse Gip promoter in entero-endocrine STC-1 cells.

Mouse Gip promoter contains functional TCF/LEF binding elements conserved in rat and humans cis-Regulatory elements targeted by WNT/lithium effectors and responsible for Gip induction remained unidentified. In silico analysis revealed nine putative TCF/LEF binding sites in the mouse Gip regulatory sequences shown schematically in Fig. 5a. The ability of putative TCF/LEF binding sites to

a

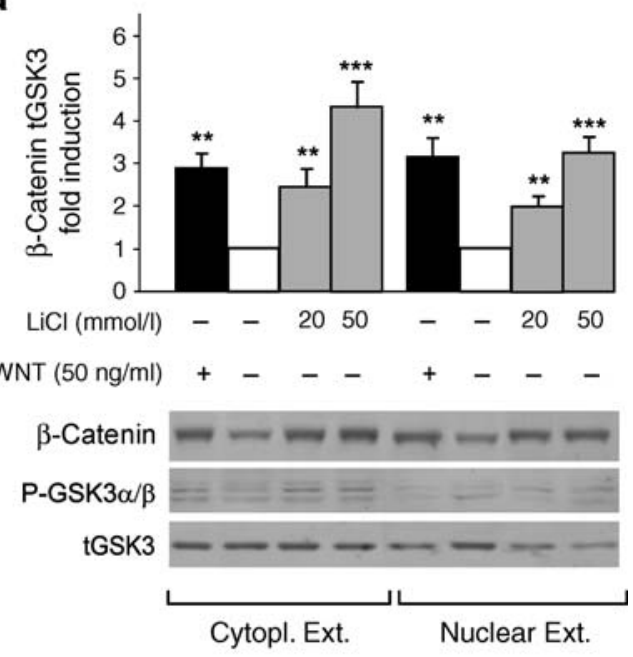

b

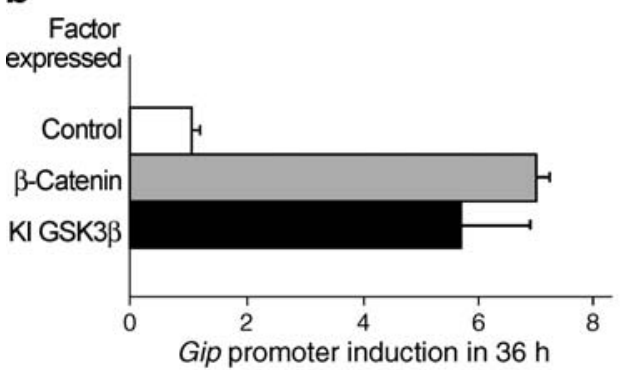

(RLU)

Fig. 4 Lithium and WNT increase soluble $\beta$-catenin, and $\beta$-catenin induces mouse Gip promoter activity in entero-endocrine cells. a Western blot analysis of cytoplasmic (Cytopl.) or nuclear (NE) extracts (Ext.) from STC-1 cells cultured for $24 \mathrm{~h}$ with $\mathrm{LiCl}$ or recombinant WNT3A at the indicated concentrations, with blot showing $\beta$-catenin, inactive P-Ser9/21 GSK $3 \alpha / \beta$ P-GSK $3 \alpha / \beta$ and total GSK3 $\alpha / \beta$ (tGSK3) as loading control, and bar graph with quantification of $\beta$-catenin induction data from three independent experiments; results are mean \pm SEM and expressed as fold induction over the control untreated cells. ${ }^{* *} p<0.01$ and $* * * p<0.0001$ according to one-way ANOVA. b Luciferase assays on STC-1 cells cotransfected with the mouse Gip promoter reporter as described in Fig. $3 b$ revealed that $\beta$-catenin overexpression induced mouse Gip promoter activity as strongly as kinase inactive (KI) GSK3 $\beta$ ( $\sim$ sixfold in $36 \mathrm{~h}$ ). Relative luciferase activities were calculated as fold induction relative to a corresponding control (mean $\pm \mathrm{SEM}, n \geq 3$ ). RLU, relative luciferase units 
bind endogenous STC-1 factors was assayed by gel retardation (EMSA) (Fig. 5b). Oligonucleotides spanning theoretical mouse Gip TCF/LEF binding sites competed with a labelled probe bearing a consensus TCF/LEF binding site for binding of STC-1 extracts. Recombinant purified glutathione $S$-transferase (GST)-tagged LEF (Recomb) or Jurkat extracts served as positive retarding controls. TCF/ LEF binding sites 1 and 2 (in the first intron), and 5 (in the proximal promoter) efficiently competed with binding of endogenous TCF/LEF factors to the consensus probe, whereas TCF/LEF binding sites 6,7 and 8 , or an unrelated oligonucleotide did not compete (oligonucleotide sequences, see ESM Table 1). Thus, at least three TCF/LEF binding sites were able to bind endogenous STC-1 factors in vitro. Systematic deletions of the $5^{\prime}$-flanking region were challenged for $\beta$-catenin-mediated activation in co-transfections. Luciferase assays were used to test their functionality (Fig. 5c, d). Both full-length and a promoter-only construct (TCF/LEF binding sites 5-9) mediated a similar seven- to tenfold induction by $\beta$-catenin. Thus, deletion of intronic sites TCF/ LEF binding sites 1 to 4 hardly affected $\beta$-catenin-mediated induction despite the strong affinity shown by the first two of these in vitro (see EMSA above). These results suggest that the main cis-element for $\beta$-catenin induction was contained in the promoter. Deletion of TCF/LEF binding sites 7 to 9 in the promoter did not affect $\beta$-catenin-dependent mouse Gip promoter induction, validating the low affinity of these sites for endogenous TCF/LEF factors as observed in EMSA. Western blotting (Fig. 5e) showed similar $\beta$ catenin levels in transfected cells. Thus, TCF/LEF binding sites 5 and/or 6 mediated $\mathrm{WNT} / \beta$-catenin induction of mouse Gip promoter. The lack of affinity shown in EMSA by the oligonucleotide containing TCF/LEF binding site 6 for endogenous TCF/LEF factors strongly suggests that binding site 5 was the functional site mediating $\beta$-catenin induction. To prove its functionality, we deleted either TCF/ LEF binding site 6 or 5 , and challenged for $\beta$-catenin induction (Fig. 5f, g). $\beta$-Catenin induction through the fragment containing only TCF/LEF binding sites $5 / 6$ reproduced induction obtained with the full-length construct. Deletion of TCF/LEF binding site 6 did not affect, while deletion of site 5 abolished $\beta$-catenin induction. We concluded that the former in the proximal promoter is the cis-element functionally responsible for $\beta$-catenin induction of the mouse Gip promoter. Western blotting (Fig. 5e, h) showed similar $\beta$ catenin levels in transfected cells. Note that $\beta$-catenindependent Gip induction varied from around fivefold in $36 \mathrm{~h}$ (Fig. 5g) up to about tenfold in $48 \mathrm{~h}$ (Fig. $5 \mathrm{~d}$ ).

The importance of TCF/LEF binding site 5 is supported by a high degree of conservation between human, rat and mouse promoter sequences (see alignment in Fig. 6a). Furthermore, oligonucleotides containing human and rat promoter TCF/LEF binding sites strongly competed in
EMSA with the consensus probe (data not shown), suggesting that $\mathrm{WNT} / \beta$-catenin regulation of GIP production occurs in mouse, rats and humans.

TCF4 and LEF1 bind to TCF/LEF binding site 5 in mouse Gip promoter and exchange co-factors in response to lithium In vivo binding of TCF/LEF factors to the TCF/ LEF binding site 5 (TL5)-containing region of mouse Gip promoter was examined by ChIP using STC-1 cells treated with or without lithium for $24 \mathrm{~h}$. Figure $6 \mathrm{~b}$ shows a scheme of mouse Gip gene indicating positions of primer pairs used. Experimental primers amplified a $302 \mathrm{bp}$ fragment spanning TCF/LEF binding site 5 (positions $-273 /+25$ in mouse Gip promoter). Negative control primers amplified a TCF/LEF binding site-free region of $300 \mathrm{bp}$ within mouse Gip intron 2. Positive control primers spanning the TCF/ LEF binding site reported at the promoter for $G c g$ (position -170) were also used. Primer pairs rendered the expected product when input chromatin was used as template (Fig. 6c). Lithium induced changes in the binding of LEF1, TCF4, $\beta$-catenin or HDAC1 to TCF/LEF binding site 5 were evaluated by semi-quantitative PCR on immunoprecipitated chromatin. Representative gels

Fig. 5 Mouse Gip promoter contains functional TCF/LEF binding elements. a Nine theoretical TCF/LEF binding sites (grey squares) were found in mouse Gip promoter and 5'-flanking untranslated region. E, exon. b EMSA using a fluorescently labelled probe containing the consensus TCF/LEF element present in the Brn2 (also known as Pou 3f2) promoter. Lane 1: free probe. Lanes 2 and 3 were positive binding controls using Jurkat extracts and recombinant (Recomb) glutathione $S$-transferase-tagged LEF respectively. Lane 4: binding of endogenous TCF/LEF factors in STC-1 extracts. Lanes 5-10: binding of STC-1 factors to the consensus was competed for with $100 \times$ excess of oligonucleotide probes containing the indicated $\mathrm{TCF} / \mathrm{LEF}$ binding sites numbered as above (a). Lane 11: positive competition control (C) where binding to the labelled BRN2 oligonucleotide was competed for with $100 \times$ excess of the same unlabelled oligonucleotide. Lane 12: negative competition control, where binding was competed for with $100 \times$ excess of an unrelated (UR) oligonucleotide. Positions of the retarded complexes of endogenous TCF/LEF or recombinant (rGST/LEF) are indicated on the left. Mouse Gip TCF/LEF binding sites 1, 2 and 5 strongly competed for binding to the consensus (lanes 5-7), while sites 6 to 8 did not show affinity for endogenous STC-1 factors (lanes 8-10). c-h Luciferase assays to test the functionality of TCF/LEF sites. $\beta$-Catenin-dependent induction of the full length (FL) 5 '-flanking region of mouse Gip gene cloned (from position -925 to +831 ) was compared with that of deletions. c Scheme of constructs transfected. Grey squares denote TCF/LEF site numbers as above (a). e, h The related western blots indicate that exogenous $\beta$-catenin levels (upper bands) were similar in all transfections and endogenous $\beta$-catenin levels (lower bands) were also similar. $\mathbf{d}$ TCF/LEF binding sites at the promoter are responsible for most of $\beta$-catenin-dependent induction. g TCF/LEF binding site 5 (TL5) alone, but not TL6 is capable of mediating $\beta$-catenin induction. Lack of $\beta$-catenin induction in cells co-transfected with an empty vector (pCMV) demonstrates that $\beta$ catenin induction occurred through specific mouse Gip promoter sequences. White bars, control; black bars, $\beta$-catenin $48 \mathrm{~h}(\mathbf{d}), \beta$ catenin $36 \mathrm{~h}(\mathrm{~g})$. RLU, relative luciferase units 


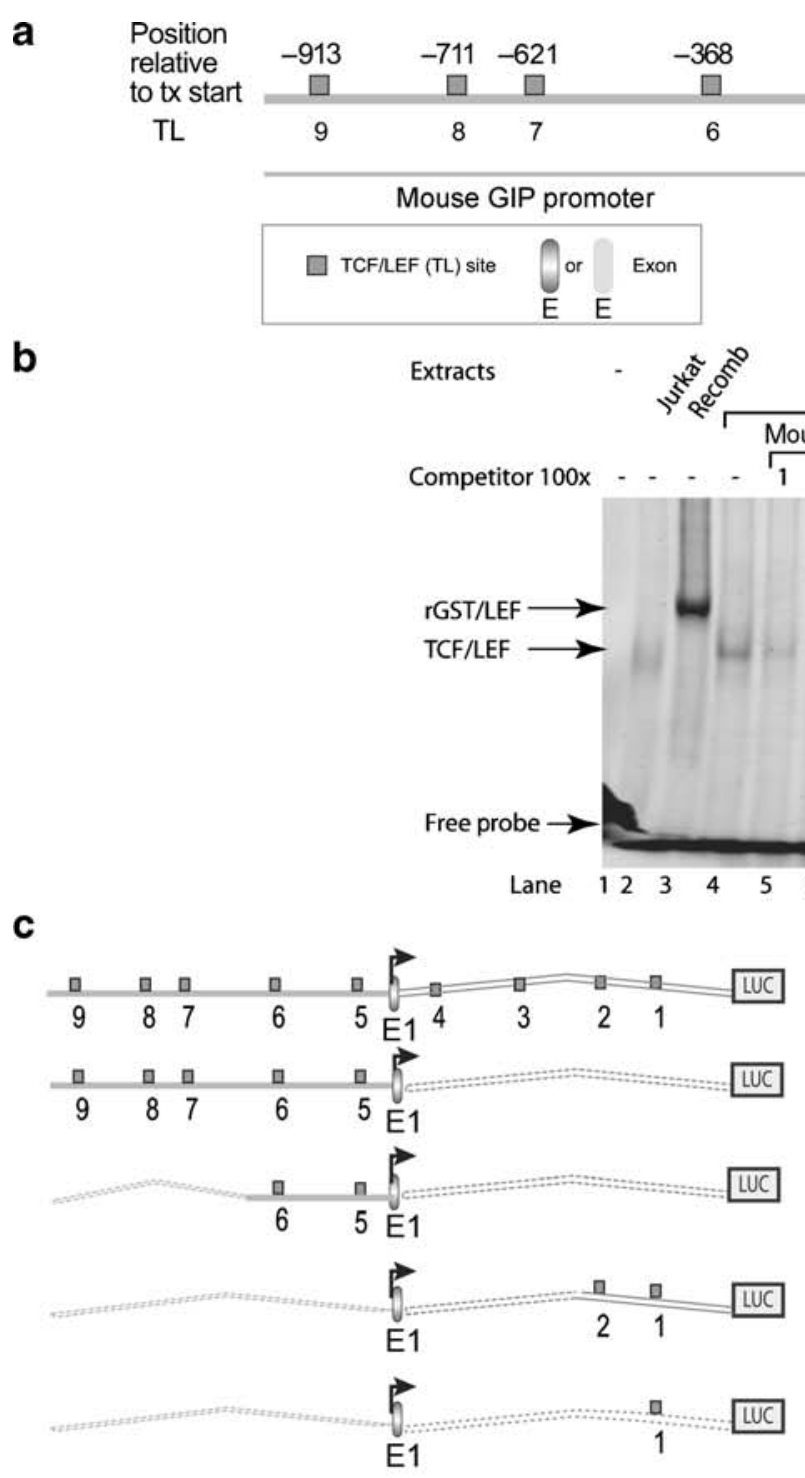

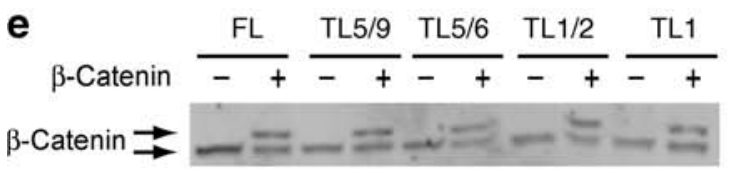

f

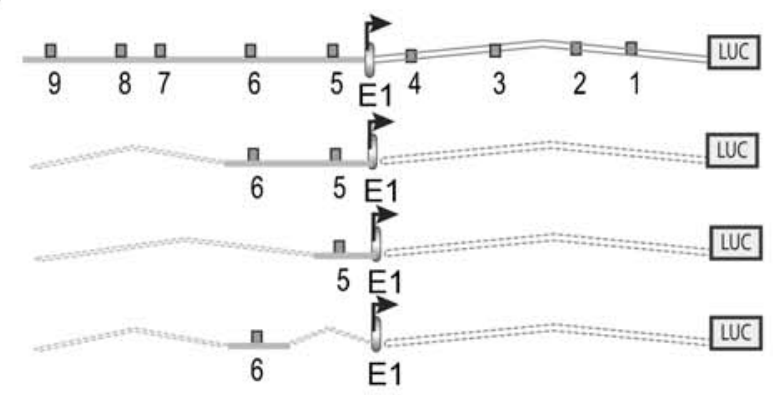

h
$\beta$-Catenin
$\beta$-Catenin $\rightarrow+$
STC-1

$\begin{array}{cccccc}\stackrel{-1}{5} & +73 & +305 & +573 & +752 & \\ E & 4 & 3 & 2 & 1 & E\end{array}$

$1^{\text {st }}$ exon + intron

Mouse GIPTCF/LEF sites

$\begin{array}{llllll}7 & 8 & 9 & 10 & 11 & 12\end{array}$

d

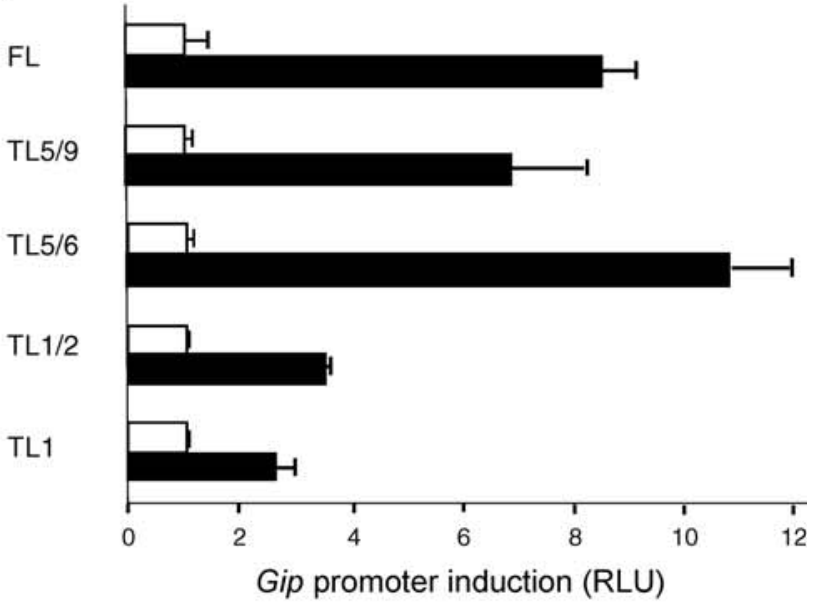

g

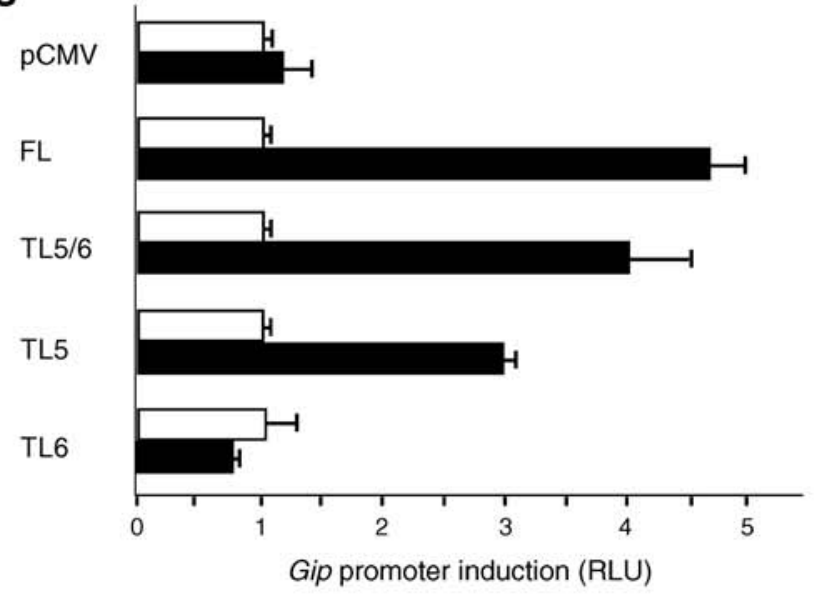


a

Gip promoter TL5 site

Mus musculus:NT_165773.2. Pos. [-138/-130]

Rattus norvegicus:gb|AC120322.5.Pos. [-138/-130]

ATACCCAAATGTTAATCACCCATTAGCACAGCCCAGGAGCAAAGGGGAAAGTGATTAGGT

1111111111111111111111111111111 11 111111111111111111111111 ATACCCAAATGTTAATCACCCATTAGCACAGGCCCAGAGCAAAGGGGAAGTGATTAGGT

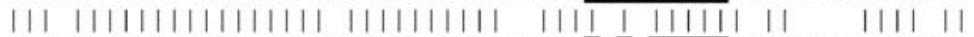

Homo sapiens:NW_001838448.1.Pos. [-141/-133]

ATAGCCAAATGTTAATCACCAATTAGCACAGTTCAGGTGGAAGGGCAACTCTATTATGT

Gcg promoter TL site

Mus musculus:NW_001030694.1 Pos. [-170/-162]

Rattus norvegicus:Nw_047655.1. Pos. [-220/-212]

TCCAAACTGCCCTTTCCATTCCCAAACAGAAA-

-GGCACAAGAGTAAATAAAATGTTTCCGGG

||||||||||||||||||||||||||||||

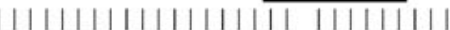

TCCAAACTACCCTTTCCATTCCCAAACAGAAA----------GGCACAAGAGTAAATAAAAAGTTTCCGGG

||||| || | || ||||||||||| || ||| | |||||||| ||||||||||||| ||

Homo sapiens:Nw 001838448.1. Pos. [-189/-181] TCCAAGCTGCTCTCTCCATTCCCAACCAAAAAAAAAAAAAAAAGATACAAGAGTGCATAAAAA_GTTTCCAGG

b

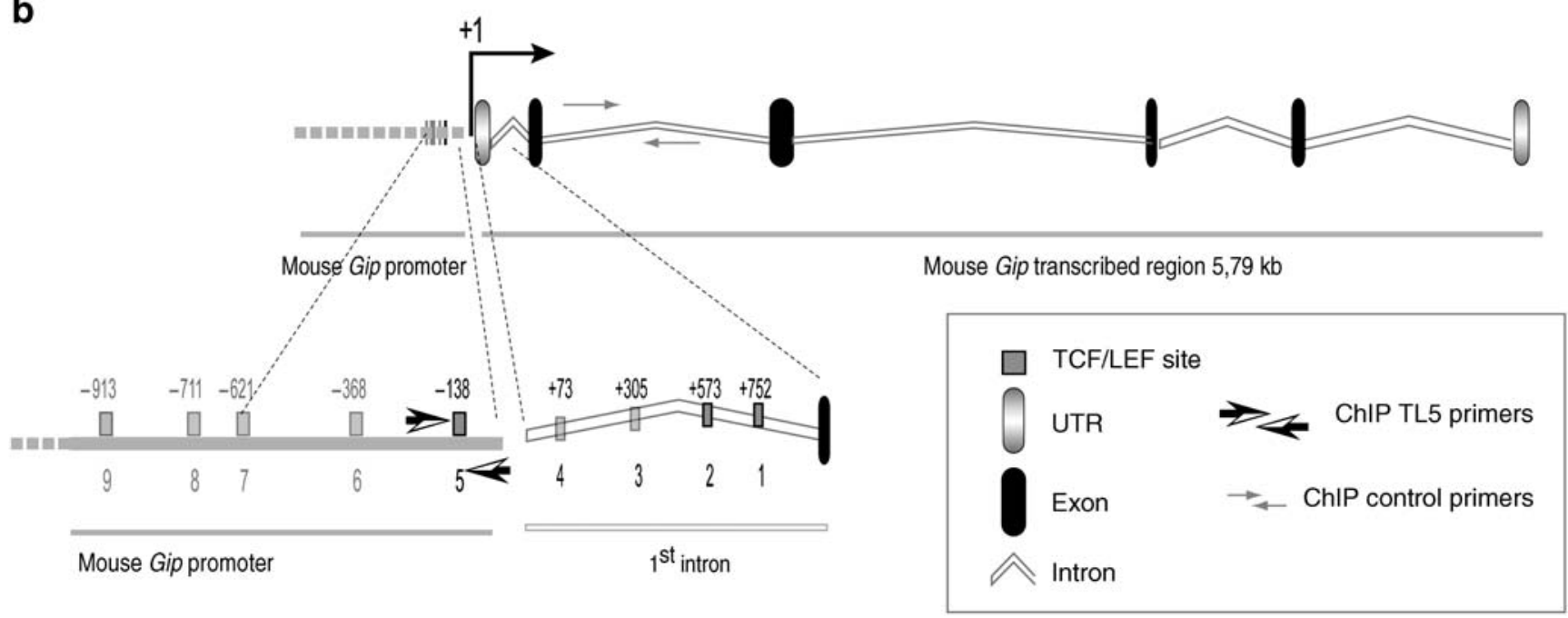

C

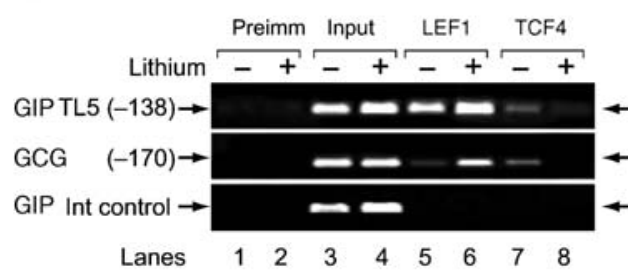

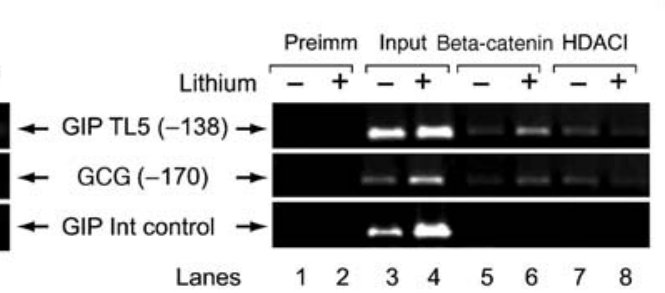

d

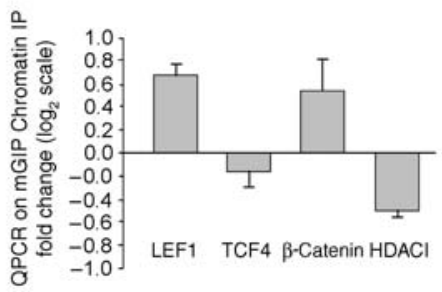

(Fig. 6c) show specific PCR fragments for mouse Gip and Gcg promoter sites. Pre-immune rabbit serum did not immunoprecipitate chromatin to render PCR fragments confirming the specificity of antibodies. Both LEF1 and TCF4 bound chromatin spanning mouse Gip TCF/LEF binding site 5 (positions $-138 /-130$ ) and the previously reported site at the $G c g$ promoter (positions $-170 /-162$ ) in the absence of lithium (Fig. 6c). Strikingly, addition of lithium diminished chromatin immunoprecipitated through TCF4 and increased LEF1-immunoprecipitated chromatin in both mouse Gip and Gcg promoter sites (Fig. 6c). This result suggests that LEF1 binding to these sites was favoured at the expense of TCF4. Thus, environmental signals such as lithium regulate binding specificity of particular TCF/LEF factors to their target sites. Anti-LEF1 or anti-TCF4 did not immunoprecipitate the TCF/LEF-free mouse Gip intron (Fig. 6c), demonstrating that binding to the mouse Gip and $\mathrm{Gcg}$ promoter sites was specific. Selection of which factor (LEF1 or TCF4) is bound to these closely related promoters (mouse Gip and Gcg) was just one chromatin remodelling output promoted by lithium, which additionally selected the co-factors $(\beta$-catenin or 
Fig. $6 \mathrm{TCF} / \mathrm{LEF}$ sites are conserved in mouse Gip and Gcg proximal promoters. TCF4/LEF1 bind in vivo to TCF/LEF binding site 5 in the mouse Gip promoter and exchange co-factors in response to lithium. a Alignment of sequences spanning TCF/LEF binding site 5 (TL5) in mouse, rat and human GIP and in proglucagon (which direct synthesis of GLP-1) promoters, spanning the previously reported TCF/LEF site. Accession numbers of sequences used are indicated. The position of the sites referred to transcription initiation are also included: [ $-138 /$ -130] for mouse Gip and [-170/-162] for mouse Gcg. b Scheme of mouse Gip gene depicting the promoter and 5'-flanking sequences with putative TCF/LEF binding sites and positions occupied, intron/ exon structure and 3' untranslated region. Sequence is based on GenBank accession number NC_000077.5. c Semiquantitative PCR on chromatin immunoprecipitated with each antibody showing that lithium increases affinity of the indicated TCF/LEF binding sites in mouse Gip and Gcg promoters for LEF1 and $\beta$-catenin as indicated. GIP TL5 (-138), mouse site at position [-138/-130]; GCG $(-170)$, mouse site in the $G c g$ promoter at position $[-170 /-162]$ as depicted in panel (a). Lanes 1 to 2, control for the specificity of the antibodies: preimmune (preimm) serum. Lanes 3 to 4 , input chromatin used as template renders the expected fragment with all three primer pairs. Lanes 5 to 6 (LEF1): lithium increased LEF1 immunoprecipitated chromatin fragments from mouse Gip and Gcg promoters spanning the indicated sites. Lanes 7 to 8 (TCF4): lithium decreased chromatin immunoprecipitated through TCF4 at the corresponding sites. The bottom gel shows that neither LEF1 nor TCF4 antibodies could immunoprecipitate chromatin containing the TCF/LEF free region at the intron. Specific ChIP was increased by lithium through $\beta$-catenin (lanes 5, 6) and decreased through HDAC1 (lanes 7, 8). d Quantitative PCR performed on five biological replicas. Normalised values obtained with lithium divided by values obtained without lithium are presented as mean \pm SEM on a $\log _{2}$ scale. Values for LEF1 and $\beta$-catenin are positive, indicating that lithium favours immunoprecipitation through these factors. Negative values for TCF4 and HDAC1 indicate that ChIP through these factors is disfavoured by lithium

HDAC) recruited to the promoter (Fig. 6c). As expected, lithium treatment favoured $\beta$-catenin binding to both promoters, displacing the co-repressor HDAC1 (Fig. 6c). Anti- $\beta$-catenin (or anti-HDAC1) immunoprecipitation of either input chromatin or chromatin previously immunoprecipitated by anti-LEF1 or anti TCF4 antibodies (data not shown) led us to the same conclusion. PCR fragments were gel purified and confirmed by sequencing to span mouse Gip TCF/LEF binding site 5 (position -138) and Gcg TCF/ LEF binding site $(-170)$. Thus, lithium remodels transcriptional complexes built upon the mouse Gip or Gcg promoters at least in two different aspects: (1) choice of the TCF/LEF factor; and (2) choice of co-factors bound. To confirm our results on mouse Gip promoter, quantitative PCR was performed in five biological replicas (Fig. 6d). The magnitude and direction of lithium-induced changes were calculated as the ratios $+\mathrm{Li}:-\mathrm{Li}$, i.e. values obtained in the presence of lithium divided by values obtained in its absence. A ratio $+\mathrm{Li}:-\mathrm{Li}=1$ means no influence of lithium, while $+\mathrm{Li}:-\mathrm{Li}>1$ or $<1$ means that lithium respectively favours or disfavours ChIP through the specified factor. Results (Fig. 6d) are presented as mean \pm SEM on a $\log _{2}$ scale because it is more intuitive: the ratio $+\mathrm{Li}:-\mathrm{Li}=1$ is transformed to value $=0$, where the $\mathrm{x}$ axis is located $\left(\log _{2}\right.$
$(1)=0)$. Values $+\mathrm{Li}:-\mathrm{Li}>1$ obtained when lithium favoured binding/immunoprecipitation appear in the positive side of the graph. Conversely $+\mathrm{Li}:-\mathrm{Li}<1$ values obtained when lithium disfavoured binding/immunoprecipitation appear in the negative side. In agreement with semiquantitative PCRs, quantitative PCR results show that LEF1 and $\beta$ catenin binding were favoured in the presence of lithium while immunoprecipitation through TCF4 and HDAC1 was diminished by lithium. These results suggest that lithium promotes replacement of TCF4/co-repressor complexes by LEF $1 / \beta$-catenin-activating complexes at mouse Gip TCF/ LEF binding site 5. Recombinant WNT3A also increased binding of both LEF1 and $\beta$-catenin to this region of the mouse Gip promoter (ESM Fig. 2)

The model in Fig. 7 summarises our understanding of the changes induced by WNT or lithium on mouse Gip and Gcg promoters: $\beta$-catenin entry into the nucleus (alone or in complex with LEF1) would result in TCF4/de-acetylases (HDAC1) exclusion, favouring LEF1/ $\beta$-catenin binding to the site. Local nucleosomes may then be acetylated by histone acetylases brought by $\beta$-catenin, allowing chromatin opening to the basal transcription machinery.

\section{Discussion}

We asked whether WNT signalling induces Gip gene expression and promotes incretin production by enteroendocrine cells. This question entails another, as yet unclear aspect of WNT signalling, i.e. whether it plays a role in differentiated entero-endocrine cells, given that GIP and GLP-1 may be considered differentiation markers for $\mathrm{K}$ and $\mathrm{L}$ intestinal cells respectively. We found that $\mathrm{WNT} / \beta$ catenin or lithium induced expression of Gip and Gcg (to produce GIP and GLP-1 respectively) to the same levels in entero-endocrine STC-1 cells. STC-1 cells are the best in vitro system for evaluating GIP production. Jin and coworkers showed lithium-induced GLP-1 synthesis in GLUTag cell lines using a chimera-containing rat G2 enhancer fused to thymidine kinase minimal promoter to direct luciferase synthesis. A TCF/LEF binding site identified was shown by ChIP to bind TCF4 and $\beta$-catenin $[17$, 18]. We obtained essentially the same results in STC-1 cells using strictly mouse promoter sequences $(\mathrm{Gcg})$ fused to luciferase. Our results reinforce those of Ni et al. [17] and generalise $\mathrm{WNT} / \beta$-catenin-dependent induction to both incretins: GIP and GLP-1. Our Gcg construct bore the mouse promoter (not rat) and lacked the thymidine kinase minimal promoter sequence (which may mask transcriptional effects), and yet we obtained inductions for $\mathrm{Gcg}$ similar to those obtained by Jin and co-workers $[17,18]$ in an alternative cell context (STC-1 cells). Beyond that, we 


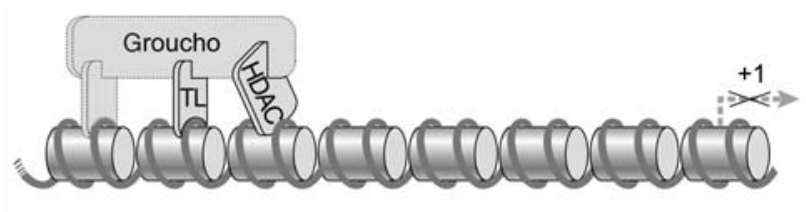

WNT

Lithium

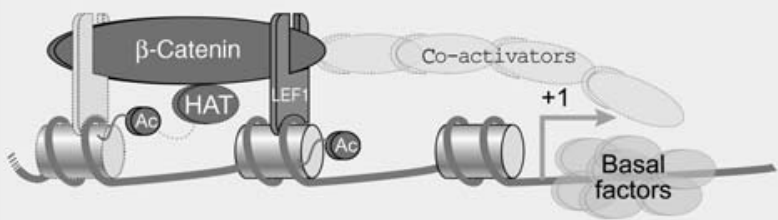

Repressed Tx

GIP/GLP-1 silenced

TCF4 or LEF1 bound

GIP/GLP-1 expressed:

LEF1/BCAT favoured

Fig. 7 Lithium or WNT/ $\beta$-catenin remodel the transcriptional complexes built upon them. Model of incretin production control by WNT signalling and lithium. In the absence of lithium or WNT signalling, TCF/LEF factors, bound at -170 and -138 in mouse Gcg and Gip promoters respectively would keep the co-repressors Groucho and HDAC1 bound, maintaining interactions between local nucleosomes. Consequently chromatin would be compacted and the genes silenced. In the presence of lithium or WNT signals soluble $\beta$-catenin increases

show a replacement of TCF4 by LEF1 and of co-repressor HDAC 1 by co-activator $\beta$-catenin at a position close to the transcription initiation site in the mouse Gip promoter. Uninduced mouse Gip and Gcg promoters bind both TCF4 and LEF1, but intriguingly lithium and WNT impose a preference for LEF1 in STC-1 cells. LEF1 is less abundant than TCF4 in entero-endocrine cells according to our own unpublished quantitative RT-PCR results (A. ChocarroCalvo, J.M. García-Martínez and C. García-Jiménez). Consequently, LEF1 selection must be based on common (architectural) changes boosted by lithium at these promoters. Lithium-induced $\beta$-catenin accumulation may promote $\beta$-catenin-LEF1 association in the cytoplasm, facilitating nuclear entry of the complex in enteroendocrine cells as previously shown in other cells [39]. Nuclear $\beta$-catenin-LEF1 complexes would replace preexisting TCF4-co-repressor complexes in these promoters. Additional $\beta$-catenin-LEF1 interactions may facilitate the selectivity and deserve further exploration. TCF4 and LEF1 bind the same DNA element and their relative affinity for $\beta$-catenin has not been compared in entero-endocrine cells treated with lithium. It seems easier, however, to bind preexisting LEF1- $\beta$-catenin complexes, displacing TCF4-corepressors from the site, than to dissociate LEF1 from $\beta$-catenin and TCF4 from co-repressors, re-associate TCF4 and export or sequester LEF1. Understanding the mechanisms behind TCF/LEF factors selectivity at promoters is one of the major challenges in the field and we are currently working to test this model. and enters the nucleus (probably in a complex with LEF1). The complex $\beta$-catenin/LEF1 displaces TCF4-co-repressors including histone deacetylases (HDACs) from the site and brings in coactivators (such as histone acetyl transferases [HAT]), which allow chromatin opening and recruitment of the transcription machinery. TL, TCF/LEF binding site; Tx, transcription; Ac, Acetylated histone tails; BCAT, $\beta$-catenin

Mechanistically, our results indicate that lithium induces incretins (GIP and GLP-1) synthesis in mouse STC-1 cells, increasing binding of the WNT effector $\beta$-catenin/LEF1 to these promoters. A high degree of interspecies TCF/LEF binding site sequence conservation in both mouse Gip and Gcg promoters (Fig. 6a) highlights architectural similarities and their importance.

Synthesis of incretins, as insulin inducers, plays a role in diabetes. Tcf7l2 variants are associated with increased type 2 diabetes risk and impaired incretin-induced insulin secretion [40], and have linked WNT with type 2 diabetes [41]. GLP-1, as inducer and effector of WNT signalling [19], links WNT, an incretin and diabetes. We show here that WNT induces incretin synthesis in general (GLP-1 and GIP). Altered GIP synthesis and signalling are linked to type 2 diabetes mainly through obesity (an engine of the diabetes epidemic) and hyperinsulinaemia. The observations detailed in the next paragraph suggest that 'hyperGIPaemia', followed by beta cell desensitising to GIP, may lie at the onset of diabetes (see below). Consequently, preventive strategies focussed on GIP should be investigated. This may complement the current therapeutic improvement offered by GLP-1 analogues. Observations linking altered GIP synthesis/signalling to obesity and diabetes come from mouse/rat models and human patients. In mouse models, GIP receptor knockout animals are resistant to diet-induced obesity and its associated risk of diabetes [9, 10, 42]. In rat models, Zucker obese rats show increased number of GIP- 
producing cells and augmented incretin-induced insulin secretion at basal glucose levels [43]. JCR:LA-cp rats, a model of hyperinsulinaemia and obesity, show more severe 'hyperGIPaemia' and hyperinsulinaemia than Zucker rats [44]. And in streptozotocin-induced diabetic rats, enhanced Gip gene expression and elevated serum levels induced chronic desensitisation of GIP receptor in vivo [2]. Finally, human diabetic and/or obese patients have increased plasma GIP levels after an oral load of glucose [45] and increased fasting responses to GIP [46, 47]. Moreover, insulinotropic effects of GIP are lost in type 2 diabetes patients and their first-degree relatives [48, 49], while GLP-1 response is conserved. This suggests a progressive failure in communication between the enteroinsular axis that includes altered GIP synthesis and function at the onset of type 2 diabetes while keeping the response to GLP-1. Our results suggest that altered WNT signalling may lie at the onset of type 2 diabetes and Tcf7l2 variants associated with diabetes may be responsible for increased LEF1 levels and incretin production, a topic that deserves further research and may lead to preventive strategies.

Finally, we would like to speculate that metabolic disorders (weight gain, obesity and type 2 diabetes) frequently found in patients chronically treated with lithium as a mood stabiliser [50] may be partially mediated by increased or untimely GIP production. GIP serum levels and responses in these patients may indicate whether modification of GIP signalling amounts to a new window of opportunity for treatment.

Thus, WNT/ $\beta$-catenin and lithium induce GIP production in mouse entero-endocrine STC-1 cells, suggesting a general role of WNT signalling and its effector, the bipartite transcription factor $\beta$-catenin-TCF/LEF, in the control of incretin production, and a novel link between WNT, incretins and diabetes.

Acknowledgements This work was financed by grant URJCCM-BIO-0522 and by Fundación Mutua Madrileña (FMM). A. Chocarro-Calvo was supported by FMM. C. M. Moya was supported by the Juan de la Cierva Programme, Spanish Ministry of Science and Innovation, and by SAF 07/60164. J. M. GarciaMartínez and C. Garcia-Jiménez are teachers at the Universidad Rey Juan Carlos (URJC), Madrid. We thank D. Hanahan (UCSF, Diabetes Center, San Francisco, CA, USA) for providing the STC-1 cells and B. Yusta (Banting and Best Diabetes Centre, Toronto, ON, Canada), P. Varga-Weisz (Babraham Institute, Cambridge, UK), M. Ros, T. Fernández-Agulló, M. García (all URJC, Madrid), P. Santisteban (IIB-CSIC, Madrid) and G. Bedó (Facultad de Ciencias, Universidad de la República, Montevideo, Uruguay) for careful reading and discussions. We also thank $\mathrm{J}$. Pérez (IIB-CSIC, Madrid) for help with the figures. Excellent technical support was provided by J. A. Mas (CAT-URJC, Madrid) and I. Velasco at our lab.

Duality of interest The authors declare that there is no duality of interest associated with this manuscript.

\section{References}

1. Gault VA, Irwin N, Harriott P, Flatt PR, O'Harte FP (2003) DPP IV resistance and insulin releasing activity of a novel disubstituted analogue of glucose-dependent insulinotropic polypeptide, (Ser2-Asp13)GIP. Cell Biol Int 27:41-46

2. Tseng CC, Boylan MO, Jarboe LA, Usdin TB, Wolfe MM (1996) Chronic desensitization of the glucose-dependent insulinotropic polypeptide receptor in diabetic rats. Am J Physiol 270:E661E666

3. Beck B (1989) Gastric inhibitory polypeptide: a gut hormone with anabolic functions. J Mol Endocrinol 2:169-174

4. Yamada Y, Seino Y (2004) Physiology of GIP - a lesson from GIP receptor knockout mice. Horm Metab Res 36:771-774

5. Hansotia T, Baggio LL, Delmeire D et al (2004) Double incretin receptor knockout (DIRKO) mice reveal an essential role for the enteroinsular axis in transducing the glucoregulatory actions of DPP-IV inhibitors. Diabetes 53:1326-1335

6. Althage MC, Ford EL, Wang S, Tso P, Polonsky KS, Wice BM (2008) Targeted ablation of glucose-dependent insulinotropic polypeptide-producing cells in transgenic mice reduces obesity and insulin resistance induced by a high fat diet. J Biol Chem 283:18365-18376

7. McClean PL, Irwin N, Cassidy RS, Holst JJ, Gault VA, Flatt PR (2007) GIP receptor antagonism reverses obesity, insulin resistance, and associated metabolic disturbances induced in mice by prolonged consumption of high-fat diet. Am J Physiol Endocrinol Metab 293:E1746-E1755

8. Miyawaki K, Yamada Y, Yano H et al (1999) Glucose intolerance caused by a defect in the entero-insular axis: a study in gastric inhibitory polypeptide receptor knockout mice. Proc Natl Acad Sci U S A 96:14843-14847

9. Yamada C, Yamada Y, Tsukiyama K et al (2007) Genetic inactivation of GIP signaling reverses aging-associated insulin resistance through body composition changes. Biochem Biophys Res Commun 364:175-180

10. Hansotia T, Maida A, Flock $G$ et al (2007) Extrapancreatic incretin receptors modulate glucose homeostasis, body weight, and energy expenditure. J Clin Invest 117:143-152

11. Meier JJ, Nauck MA, Schmidt WE, Gallwitz B (2002) Gastric inhibitory polypeptide: the neglected incretin revisited. Regul Pept 107:1-13

12. Welters HJ, Kulkarni RN (2008) Wnt signaling: relevance to betacell biology and diabetes. Trends Endocrinol Metab 19:349-355

13. Clevers $H$ (2006) Wnt/beta-catenin signaling in development and disease. Cell 127:469-480

14. Roose J, Clevers H (1999) TCF transcription factors: molecular switches in carcinogenesis. Biochim Biophys Acta 1424:M23M37

15. Klein PS, Melton DA (1996) A molecular mechanism for the effect of lithium on development. Proc Natl Acad Sci U S A 93:8455-8459

16. Hedgepeth CM, Conrad LJ, Zhang J, Huang HC, Lee VM, Klein PS (1997) Activation of the Wnt signaling pathway: a molecular mechanism for lithium action. Dev Biol 185:82-91

17. Ni Z, Anini Y, Fang X, Mills G, Brubaker PL, Jin T (2003) Transcriptional activation of the proglucagon gene by lithium and beta-catenin in intestinal endocrine $\mathrm{L}$ cells. J Biol Chem 278:1380-1387

18. Yi F, Brubaker PL, Jin T (2005) TCF-4 mediates cell type-specific regulation of proglucagon gene expression by beta-catenin and glycogen synthase kinase-3beta. J Biol Chem 280:1457-1464

19. Gustafson B, Smith U (2008) WNT signalling is both an inducer and effector of glucagon-like peptide-1. Diabetologia 51:17681770 
20. Boylan MO, Jepeal LI, Jarboe LA, Wolfe MM (1997) Cellspecific expression of the glucose-dependent insulinotropic polypeptide gene in a mouse neuroendocrine tumor cell line. $\mathrm{J}$ Biol Chem 272:17438-17443

21. Jepeal LI, Fujitani Y, Boylan MO, Wilson CN, Wright CV, Wolfe MM (2005) Cell-specific expression of glucose-dependentinsulinotropic polypeptide is regulated by the transcription factor PDX-1. Endocrinology 146:383-391

22. Jepeal LI, Boylan MO, Michael Wolfe M (2008) GATA-4 upregulates glucose-dependent insulinotropic polypeptide expression in cells of pancreatic and intestinal lineage. Mol Cell Endocrinol 287:20-29

23. Fujita Y, Chui JW, King DS et al (2008) Pax6 and Pdx1 are required for production of glucose-dependent insulinotropic polypeptide in proglucagon-expressing L cells. Am J Physiol Endocrinol Metab 295:E648-E657

24. Pinto D, Gregorieff A, Begthel H, Clevers H (2003) Canonical Wnt signals are essential for homeostasis of the intestinal epithelium. Genes Dev 17:1709-1713

25. Wang Y, Giel-Moloney M, Rindi G, Leiter AB (2007) Enteroendocrine precursors differentiate independently of Wnt and form serotonin expressing adenomas in response to active beta-catenin. Proc Natl Acad Sci U S A 104:11328-11333

26. Rindi G, Grant SG, Yiangou Y et al (1990) Development of neuroendocrine tumors in the gastrointestinal tract of transgenic mice. Heterogeneity of hormone expression. Am J Pathol 136:1349-1363

27. Schug J (2008) Using TESS to predict transcription factor binding sites in DNA sequence. Curr Protoc Bioinformatics Chapter 2: Unit 2.6

28. Munoz-Montano JR, Lim F, Moreno FJ, Avila J, Diaz-Nido J (1999) Glycogen synthase kinase-3 modulates neurite outgrowth in cultured neurons: possible implications for neurite pathology in Alzheimer's disease. J Alzheimers Dis 1:361-378

29. Andrews NC, Faller DV (1991) A rapid micropreparation technique for extraction of DNA-binding proteins from limiting numbers of mammalian cells. Nucleic Acids Res 19:2499

30. Goodall J, Martinozzi S, Dexter TJ et al (2004) Brn-2 expression controls melanoma proliferation and is directly regulated by betacatenin. Mol Cell Biol 24:2915-2922

31. Livak KJ, Schmittgen TD (2001) Analysis of relative gene expression data using real-time quantitative PCR and the 2(-Delta Delta C(T)) Method. Methods 25:402-408

32. Jope RS (2003) Lithium and GSK-3: one inhibitor, two inhibitory actions, multiple outcomes. Trends Pharmacol Sci 24:441-443

33. Ryves WJ, Harwood AJ (2001) Lithium inhibits glycogen synthase kinase- 3 by competition for magnesium. Biochem Biophys Res Commun 280:720-725

34. Wada A, Yokoo H, Yanagita T, Kobayashi H (2005) Lithium: potential therapeutics against acute brain injuries and chronic neurodegenerative diseases. J Pharmacol Sci 99:307-321

35. Yanagita T, Maruta T, Uezono $Y$ et al (2007) Lithium inhibits function of voltage-dependent sodium channels and catechol- amine secretion independent of glycogen synthase kinase-3 in adrenal chromaffin cells. Neuropharmacology 53:881-889

36. Liang MH, Wendland JR, Chuang DM (2008) Lithium inhibits Smad3/4 transactivation via increased CREB activity induced by enhanced PKA and AKT signaling. Mol Cell Neurosci 37:440453

37. Korinek V, Barker N, Morin PJ et al (1997) Constitutive transcriptional activation by a beta-catenin-Tcf complex in APC -/- colon carcinoma. Science 275:1784-1787

38. Veeman MT, Slusarski DC, Kaykas A, Louie SH, Moon RT (2003) Zebrafish prickle, a modulator of noncanonical Wnt $/ \mathrm{Fz}$ signaling, regulates gastrulation movements. Curr Biol 13:680685

39. Asally M, Yoneda Y (2005) Beta-catenin can act as a nuclear import receptor for its partner transcription factor, lymphocyte enhancer factor-1 (lef-1). Exp Cell Res 308:357-363

40. Lyssenko V, Lupi R, Marchetti P et al (2007) Mechanisms by which common variants in the TCF7L2 gene increase risk of type 2 diabetes. J Clin Invest 117:2155-2163

41. Florez JC, Jablonski KA, Bayley N et al (2006) TCF7L2 polymorphisms and progression to diabetes in the Diabetes Prevention Program. N Engl J Med 355:241-250

42. Miyawaki K, Yamada Y, Ban N et al (2002) Inhibition of gastric inhibitory polypeptide signaling prevents obesity. Nat Med 8:738-742

43. Chan CB, Pederson RA, Buchan AM, Tubesing KB, Brown JC (1984) Gastric inhibitory polypeptide (GIP) and insulin release in the obese Zucker rat. Diabetes 33:536-542

44. Pederson RA, Campos RV, Buchan AM, Chisholm CB, Russell JC, Brown JC (1991) Comparison of the enteroinsular axis in two strains of obese rat, the fatty Zucker and the JCR:LA-corpulent. Int J Obes 15:461-470

45. Brown JC, Otte S (1979) Clinical studies with gastric inhibitory polypeptide. World J Surg 3:553-558

46. Jones IR, Owens DR, Luzio S, Hayes TM (1989) Glucose dependent insulinotropic polypeptide (GIP) infused intravenously is insulinotropic in the fasting state in type 2 (non-insulin dependent) diabetes mellitus. Horm Metab Res 21:23-26

47. Jones IR, Owens DR, Luzio S, Williams S, Hayes TM (1989) The glucose dependent insulinotropic polypeptide response to oral glucose and mixed meals is increased in patients with type 2 (noninsulin-dependent) diabetes mellitus. Diabetologia 32:668-677

48. Knop FK, Vilsboll T, Hojberg PV et al (2007) The insulinotropic effect of GIP is impaired in patients with chronic pancreatitis and secondary diabetes mellitus as compared to patients with chronic pancreatitis and normal glucose tolerance. Regul Pept 144:123130

49. Meier JJ, Hucking K, Holst JJ, Deacon CF, Schmiegel WH, Nauck MA (2001) Reduced insulinotropic effect of gastric inhibitory polypeptide in first-degree relatives of patients with type 2 diabetes. Diabetes 50:2497-2504

50. Kim B, Kim SJ, Son JI, Joo YH (2008) Weight change in the acute treatment of bipolar I disorder: a naturalistic observational study of psychiatric inpatients. J Affect Disord 105:45-52 